\title{
Telomere Syndrome
}

National Cancer Institute

\section{Source}

National Cancer Institute. Telomere Syndrome. NCI Thesaurus. Code C152065.

A group of disorders caused by mutation(s) that disrupt the maintenance of telomeres, resulting in the short telomere defect. 